\title{
Ostéonécrose du prémaxillaire consécutive à une coagulation intravasculaire disséminée : à propos d'un cas
}

\author{
A. Mouraret (Nancy), E. Gerard (Ars-Laquenexy), J. Le Gall (Ars-Laquenexy), R. Curien \\ (Ars-Laquenexy)
}

La coagulation intravasculaire disséminée (CIVD) est une pathologie rare correspondant à l'activation systémique de la cascade de coagulation. Les thrombi fibrineux formés, auxquels s'ajoute un éventuel vasospasme, conduisent à l'ischémie et à la nécrose des tissus. La CIVD est fréquemment associée à un traumatisme, une blessure, une complication obstétrique ou à un choc septique. De nombreux cas d'ostéonécrose dus à une CIVD ont été décrits dans la littérature orthopédique (principalement concernant la tête fémorale), mais très peu de cas sont retrouvés concernant la région maxillo-mandibulaire. Une patiente de 83 ans se présente en consultation pour l'apparition récente de mobilités au niveau du bloc incisivo-canin maxillaire. L'interrogatoire révèle le diagnostic deux mois auparavant d'une leucémie myéloïde aigue, découverte de manière fortuite suite à un choc septique à E. Coli. Les bilans biologiques réalisés au moment du sepsis étaient en faveur d'une coagulation intravasculaire disséminée (CIVD). Quelques jours après, la patiente se plaignait de douleurs et de mobilités anormales localisées sur les dents antérieures maxillaires. Les examens cliniques et radiographiques (orthopantomogramme et cone beam) objectivent une nécrose osseuse du prémaxillaire limitée par les canines, avec un séquestre indépendant du reste du maxillaire. L'étude d'imputabilité conclut à la responsabilité de la CIVD en l'absence d'autres facteurs déclenchants et en raison d'une chronologie concordante. Le diagnostic de nécrose consécutive à une CIVD est alors posé. La prise en charge consiste en une séquestrotomie sous anesthésie générale et fermeture muqueuse. Un suivi régulier est mis en place avec une évolution favorable. Le prémaxillaire possède une vascularisation très riche assurée par les branches terminales de l'artère carotide externe, et par l'artère maxillaire. Les nécroses avasculaires sont par conséquent rares dans cette région. Cette vascularisation abondante explique le peu de cas décrits dans la littérature. On retrouve deux cas de nécrose consécutive à une CIVD au niveau de la mandibule et deux cas pour le prémaxillaire. 